\title{
Strapdown Sculling Velocity Algorithms Using Novel Input Combinations
}

\author{
Lei Huang $\mathbb{D}^{1,2,3}$ Zhaochun Li $\mathbb{D}^{1},{ }^{1}$ Fei Xie $\mathbb{D},{ }^{2,4}$ and Kai Feng ${ }^{1}$ \\ ${ }^{1}$ School of Mechanical and Electrical Engineering, Nanjing Forestry University, Nanjing 210037, China \\ ${ }^{2}$ Jiangsu Province 3D Printing Equipment and Manufacturing Key Lab, Nanjing Normal University, Nanjing 210042, China \\ ${ }^{3}$ School of Instrument Science and Engineering, Southeast University, Nanjing 210096, China \\ ${ }^{4}$ School of Electrical and Automation Engineering, Nanjing Normal University, Nanjing 210042, China
}

Correspondence should be addressed to Lei Huang; huanglei@njfu.edu.cn and Fei Xie; xiefei@njnu.edu.cn

Received 19 June 2018; Accepted 16 October 2018; Published 28 October 2018

Academic Editor: Federica Caselli

Copyright (C) 2018 Lei Huang et al. This is an open access article distributed under the Creative Commons Attribution License, which permits unrestricted use, distribution, and reproduction in any medium, provided the original work is properly cited.

\begin{abstract}
Sculling motion is a standard input to evaluate the performance of the velocity algorithm in a highly dynamic environment. Conventional sculling algorithms usually adopt incremental angle/specific force increments or angular rate/specific force as algorithm inputs. However modern inertial sensors have different output types now, which do not correspond to the inputs of those traditional algorithms. For example, some inertial sensors have the integrated angular rate (incremental angle)/specific force outputs or angular rate/specific force increments outputs. Hence the conventional sculling algorithms cannot be easily applied to these situations. A novel sculling algorithm using incremental angle/specific force inputs or angular rate/specific force increments inputs is developed in this paper. The advantage of the novel algorithm is that it can calculate the carrier velocity directly without converting the dimension of inertial sensor outputs values. Theoretical analysis, digital simulations, and a trial study are carried out to verify our algorithm. The results demonstrate that for corresponding types of strapdown inertial navigation systems (SINS) the novel sculling algorithm exhibits better performance than the conventional sculling velocity algorithms.
\end{abstract}

\section{Introduction}

Improvements in maneuverability of flight vehicles necessitate SINS capable of efficiency in highly dynamic environments. To improve the precision of SINS, sculling motion is usually employed as a standard input to evaluate the performance of the velocity algorithm in a dynamic environment. Savage provided an analytical description of sculling motion in two forms $[1,2]$. In that study a digital algorithm capable of calculating the sculling integral term was described. An analytical model for the evaluation of error build-up under band-limited random process input for the digital integration algorithm was developed in a later study [3]. It was demonstrated that the digital integration process introduced a random walk type error in the output that was directly proportional to the root-mean-square input amplitude, directly proportional to the square-root of the input bandwidth, and inversely proportional to the digital integration update frequency. Ignagni derived a class of optimized sculling algorithms and demonstrated a duality between the derived class of sculling algorithms and a previously derived class of coning algorithms [4]. Kelly developed a generic equivalency between coning and sculling integrals and algorithms. The equivalency allows a coning algorithm based on incremental angle input to be converted to its corresponding sculling algorithm using a simple mathematical formula [5]. In [6] two alternative approaches were developed for deriving strapdown navigation sculling algorithms. A key point of the two approaches is the use of additional gyro/accelerometer output signals which are the increments of the angular rate/specific force multiple integrals over the iteration interval. However, all these conventional velocity algorithms adopt incremental angle/specific force increments or angular rate/specific force as algorithm inputs. However modern inertial sensors produce different types of output now. For example, certain fiber optic gyros (FOGs) and the microelectromechanical systems (MEMS) gyros have angular rate sampled output, but the laser gyro usually has integrated angular rate output. The 
quartz accelerometer output is usually specific force, but some other type of accelerometer output carrier's specific force increments. If the SINS use a gyro with an output of angular rate and an accelerometer with an output of specific force increments, these conventional sculling velocity algorithms are no longer very applicable. To solve this problem, a novel sculling algorithm using incremental angle/specific force inputs or angular rate/specific force increments inputs is developed in this paper. The novel algorithm can calculate out the carrier velocity directly without converting the dimension of inertial sensor outputs values. Owing to this, the precision of the algorithm is considerably high.

\section{Conventional Sculling Algorithms}

Velocity rate equation is

$$
\dot{V}=C_{b}^{n} f^{b}-\left(2 \omega_{i e}^{n}+\omega_{e n}^{n}\right) \times V^{n}+g^{n}
$$

The carrier velocity in navigation coordinates at time $t_{m}$ is then obtained as the integral of (1) from time $t_{m-1}$, evaluated at time $t_{m}$ as [1]

$$
\begin{aligned}
V_{m}^{n}= & V_{m-1}^{n}+C_{b(m-1)}^{n(m-1)} \int_{t_{m-1}}^{t_{m}} C_{b(t)}^{b(m-1)} f^{b} d t \\
& \quad+\int_{t_{m-1}}^{t_{m}}\left[g^{n}-\left(2 \omega_{i e}^{n}+\omega_{e n}^{n}\right) \times V_{m-1}^{n}\right] d t \\
= & V_{m-1}^{n}+C_{m-1} \Delta V_{s f m}+\Delta V_{g / c o r n}
\end{aligned}
$$

where $m$ is the computer cycle index, $\Delta V_{s f m}$ is the integrated transformed specific force increment, and $\Delta V_{g / c o r n}$ is the gravity/Carioles velocity increment. Calculating $\Delta V_{g / c o r n}$ is a relatively simple process and is therefore omitted. $\Delta V_{s f m}$ can be expanded as [5]

$$
\begin{aligned}
\Delta V_{s f m}= & \Delta V_{m}+\frac{1}{2} \Delta \theta_{m} \times \Delta V_{m} \\
& +\frac{1}{2} \int_{t_{m-1}}^{t_{m}}[\Delta \theta(t) \times f(t)+\Delta V(t) \times \omega(t)] d t \\
= & \Delta V_{m}+\Delta V_{\text {rotm }}+\Delta V_{\text {sculm }}
\end{aligned}
$$

where

$$
\begin{aligned}
\Delta V(t) & =\int f d t \\
\Delta V_{m} & =\int_{t_{m-1}}^{t_{m}} f d t \\
\Delta \theta(t) & =\int \omega d t
\end{aligned}
$$

In (3) $\Delta V_{\text {rotm }}$ is the velocity rotation correction and $\Delta V_{\text {sculm }}$ is the sculling correction. $\Delta V_{\text {sculm }}$ in (3) is a continuous integral form. The discrete algorithm for $\Delta V_{s c u l m}$, which is usually referred to as sculling algorithm, can be obtained from the coning algorithm using a simple duality formula $[4,5,7]$. For example, the 2 -interval optimal sculling algorithm using incremental angle/specific force increments inputs is $[5,8-10]$

$$
\Delta \widehat{V}_{\text {sculm }}=\frac{2}{3}\left(\Delta \theta_{1} \times \Delta V_{2}+\Delta V_{1} \times \Delta \theta_{2}\right)
$$

Its coning algorithm counterpart, namely, the 2-interval optimal coning algorithm using incremental angle input, is [11]

$$
\Delta \Phi=\frac{2}{3}\left(\Delta \theta_{1} \times \Delta \theta_{2}\right)
$$

The 2-interval optimal sculling algorithm using angular rate/specific force inputs is $[12,13]$

$$
\begin{aligned}
\Delta \widehat{V}_{\text {sculm }}= & \frac{1}{180}\left(\omega_{1} \times f_{3}+f_{1} \times \omega_{3}\right) H^{2} \\
& +\frac{7}{45}\left(\omega_{1} \times f_{2}+f_{1} \times \omega_{2}\right) H^{2}
\end{aligned}
$$

where $H$ is the attitude and velocity update period $H=$ $t_{m}-t_{m-1}$. Its coning algorithm counterpart using angular rate input is [14]

$$
\Delta \Phi=\frac{1}{45}\left(\omega_{1} \times \omega_{3}\right) h^{2}+\frac{28}{45}\left(\omega_{1} \times \omega_{2}\right) h^{2}
$$

where $h$ is the algorithm iteration interval. In a 2 -interval algorithm there is $H=2 h$.

\section{Sculling Algorithm Using Incremental Angle/Specific Force Input}

If the inertial sensors produce integrated angular rate (incremental angle)/specific force outputs, the conventional sculling algorithms such as (5) and (7) cannot calculate out the carrier's velocity directly. The velocity algorithm must include a step for conversion of integrated angular rate (incremental angle) into angular rate by digital differentiation in order to use (7) or else convert specific force into specific force increments by digital integration in order to use (5). These steps will increase computational error. To solve this problem we have developed a novel sculling algorithm using incremental angle/specific force input.

3.1. Generalized Sculling Algorithm. For a 2-interval sculling velocity algorithm, there are two sample gyro outputs $\Delta \theta_{i}$ $(i=1,2)$ at time $t_{m-1 / 2}, t_{m}$, and three sample accelerometer outputs $f_{j}(j=1,2,3)$ at times $t_{m-1}, t_{m-1 / 2}$, and $t_{m}$ over an update period $\left(t_{m-1}, t_{m}\right)$. Assume that the incremental angle $\Delta \theta$ and specific force $f$ can be approximated by the secondorder polynomial:

$$
\begin{aligned}
\Delta \theta & =a\left(t-t_{m-1}\right)+b\left(t-t_{m-1}\right)^{2} \\
f & =A+2 B\left(t-t_{m-1}\right)+3 C\left(t-t_{m-1}\right)^{2}
\end{aligned}
$$

where $a, b, A, B$, and $C$ are the polynomials coefficients. Then

$$
\begin{aligned}
a H & =3 \Delta \theta_{1}-\Delta \theta_{2} \\
b H^{2} & =2\left(\Delta \theta_{2}-\Delta \theta_{1}\right), \\
A & =f_{1}
\end{aligned}
$$




$$
\begin{aligned}
B H & =\frac{1}{2}\left(-3 f_{1}+4 f_{2}-f_{3}\right) \\
C H^{2} & =\frac{2}{3}\left(f_{1}-2 f_{2}+f_{3}\right)
\end{aligned}
$$

Substituting (9) into $\Delta V_{\text {sculm }}$ given by (3) gives

$$
\begin{aligned}
\Delta \widehat{V}_{\text {sculm }}= & \frac{1}{2} \int_{t_{m-1}}^{t_{m}}[\Delta \theta(t) \times f(t)+\Delta V(t) \times \omega(t)] d t \\
= & \frac{1}{6} A \times b H^{3}+\frac{1}{6} a \times B H^{3}+\frac{1}{4} a \times C H^{4}+\frac{1}{10} b \\
& \times C H^{5}
\end{aligned}
$$

Substituting (10) into (11) gives

$$
\begin{aligned}
\Delta \widehat{V}_{\text {sculm }}= & -\frac{H}{20} \Delta \theta_{1} \times f_{1}-\frac{7 H}{60} \Delta \theta_{2} \times f_{1}+\frac{4 H}{15} \Delta \theta_{1} \\
& \times f_{2}-\frac{4 H}{15} \Delta \theta_{2} \times f_{2}+\frac{7 H}{60} \Delta \theta_{1} \times f_{3} \\
& +\frac{H}{20} \Delta \theta_{2} \times f_{3}
\end{aligned}
$$

This is the 2-interval generalized sculling algorithm using incremental angle/specific force input.

3.2. Optimal Sculling Algorithm. A typical sculling motion is defined as [15]

$$
\begin{aligned}
& \omega=b \Omega \cos (\Omega t) J, \\
& f=c \sin (\Omega t) K
\end{aligned}
$$

where $b$ is the amplitude of the angular vibration, $c$ is the amplitude of the specific force vibration, $J, K$ are the unit vectors along the two body axes $(y, z)$ about which the oscillations are occurring, and $\Omega$ is the frequency associated with the angular and specific force oscillations.

The incremental angle and specific force increments of the sculling motion are

$$
\begin{gathered}
\Delta \theta=\int_{t_{m-1}}^{t} \omega d t=b\left(\sin \Omega t-\sin \Omega t_{m-1}\right) J \\
\Delta V=\int_{t_{m-1}}^{t} f d t=-\frac{c}{\Omega}\left(\cos \Omega t-\cos \Omega t_{m-1}\right) K
\end{gathered}
$$

Substituting (13) and (14) into the $\Delta V_{\text {sculm }}$ term in (3) yields the truth sculling correction:

$$
\Delta V_{\text {sculm }}=\frac{b c}{2}\left(H-\frac{1}{\Omega} \sin \Omega H\right)
$$

As is seen in (15), in a sculling environment the true sculling correction $\Delta V_{\text {sculm }}$ is a constant value. For a 2interval system, the outputs of inertial sensors over a velocity update period are

$$
\begin{aligned}
\Delta \theta_{i} & =b\left[\sin \left(\Omega t_{m-1}+\frac{i}{2} \Omega H\right)\right. \\
& \left.-\sin \left(\Omega t_{m-1}+\frac{i-1}{2} \Omega H\right)\right] J, \quad i=1,2 \\
f_{j} & =c \sin \left[\Omega\left(t_{m-1}+\frac{j-1}{2} H\right)\right] K, \quad j=1,2,3
\end{aligned}
$$

The generalized form of the sculling correction using incremental angle/specific input can be given as

$$
\Delta \widehat{V}_{\text {sculm }}=\sum_{j=i+1}^{N} \sum_{i=1}^{N-1} k_{i j}\left(\Delta \theta_{i} \times f_{j}-\Delta \theta_{j} \times f_{i}\right) H,
$$

$$
N=2
$$

where $N$ is the number of iteration intervals over the velocity update period. For a 2-interval algorithm, $N=2$. It follows from (16) that

$$
\begin{aligned}
& \left(\Delta \theta_{i} \times f_{j}-\Delta \theta_{j} \times f_{i}\right) H \\
& =b c h \sin \frac{\Omega H}{4}\left[\sin \left(\frac{2 j-2 i+1}{4} \Omega H\right)\right. \\
& \left.-\sin \left(\frac{2 i-2 j+1}{4} \Omega H\right)\right]=b c h \sin \frac{\Omega H}{2} \\
& \cdot \sin \left(\frac{j-i}{2} \Omega H\right)
\end{aligned}
$$

Obviously, the sculling correction $\Delta V_{\text {sculm }}$ is only determined by $|j-i|$. Therefore (17) can be simplified as

$$
\Delta \widehat{V}_{\text {sculm }}=\sum_{j=2}^{N} k_{j-1}\left(\Delta \theta_{1} \times f_{j}-\Delta \theta_{j} \times f_{1}\right) H
$$

For a 2-interval sculling algorithm, $N=2$. Substituting (18) into (19) gives

$$
\Delta \widehat{V}_{\text {sculm }}=k_{1} b \operatorname{ch} \sin ^{2} \frac{\Omega H}{2}
$$

Sculling correction $\Delta \widehat{V}_{\text {sculm }}$ should be equal to the true sculling correction $\Delta V_{\text {sculm }}, \Delta \widehat{V}_{\text {sculm }}=\Delta V_{\text {sculm }}$. Using (19) and (15), the simultaneous equation for the unknown parameter $k$ can be obtained. Then the equation can be simplified by Taylor expanding " $\Omega H$ ":

$$
\begin{array}{ccc}
\Omega^{2} H^{3}: & \Delta \widehat{V}_{\text {sculm }} & \Delta V_{\text {sculm }} \\
& \frac{k_{1}}{4} b c & \frac{1}{12} b c
\end{array}
$$

The solution for $\Delta \widehat{V}_{\text {sculm }}=\Delta V_{\text {sculm }}$ is $k_{1}=1 / 3$. Hence the 2-interval optimal sculling algorithm using incremental angle/specific force input is derived:

$$
\Delta \widehat{V}_{\text {sculm }}=\frac{1}{3}\left(\Delta \theta_{1} \times f_{2}-\Delta \theta_{2} \times f_{1}\right) H
$$


The per-unit time algorithm error is

$$
\delta \widehat{V}_{\text {sculm }}=\left|\widehat{V}_{\text {sculm }}-V_{\text {sculm }}\right|=\frac{b c}{360}(\Omega H)^{4}
$$

In (2) and (3), the integrated transformed specific force increment $\Delta V_{s f m}$ is composed of $\Delta V_{\text {sculm }}, \Delta V_{\text {rotm }}$, and $\Delta V_{m}$. $\Delta V_{m}$ and $\Delta \theta_{m}$ in (3) can be calculated by digital integration:

$$
\begin{aligned}
& \Delta \widehat{\theta}_{m}=\Delta \theta_{1}+\Delta \theta_{2}, \\
& \Delta \widehat{V}_{m}=\left(\frac{f_{1}}{6}+\frac{4 f_{2}}{6}+\frac{f_{3}}{6}\right) H
\end{aligned}
$$

Substituting (24) into (3) gives

$$
\Delta \widehat{V}_{\text {rotm }}=\frac{1}{2}\left(\Delta \theta_{1}+\Delta \theta_{2}\right) \times\left(\frac{f_{1}}{6}+\frac{4 f_{2}}{6}+\frac{f_{3}}{6}\right) H
$$

Substituting (22), (24), and (25) into (3) gives the integrated transformed specific force increments:

$$
\begin{aligned}
\Delta \widehat{V}_{s f m}= & \left(\frac{f_{1}}{6}+\frac{4 f_{2}}{6}+\frac{f_{3}}{6}\right) H+\frac{1}{2}\left(\Delta \theta_{1}+\Delta \theta_{2}\right) \\
& \times\left(\frac{f_{1}}{6}+\frac{4 f_{2}}{6}+\frac{f_{3}}{6}\right) H \\
& +\frac{1}{3}\left(\Delta \theta_{1} \times f_{2}-\Delta \theta_{2} \times f_{1}\right) H
\end{aligned}
$$

As described previously, $\Delta V_{g / c o r n}$ in (2) can be calculated out easily; indeed it can be calculated approximatively as a constant during one update period and omitted here. $V_{m-1}^{n}$ in (2), i.e., the carrier velocity at $t_{m-1}$, has been calculated out during the last algorithm update period. Thus the carrier velocity at time $t_{m}$ can be calculated out by substituting (26) into (2).

3.3. Formalized Optimal Sculling Algorithm. For an $N$-interval sculling velocity algorithm using incremental angle/spe- cific force input, the sample number of the accelerometer outputs (specific force) is $N+1$, and the number of the gyro outputs (integrated angular rate) is $N$. The gyro/accelerometer outputs in a sculling environment defined by (13) are

$$
\begin{aligned}
& \Delta \theta_{i}=2 b \sin \frac{\Omega H}{4} \cos \Omega\left(t_{m-1}+\frac{2 i-1}{2 N} H\right) J, \\
& i=1,2, \ldots, N \\
& f_{j}=c \sin \left[\Omega\left(t_{m-1}+\frac{j-1}{N} H\right)\right] K, \\
& j=1,2, \ldots N+1
\end{aligned}
$$

As previously mentioned, $N$-interval sculling velocity algorithm can be formulated as (19). Substituting (27) into (19) we obtain

$$
\Delta \widehat{V}_{\text {sculm }}=b c H \sum_{i=1}^{N-1} k_{i} \sin \frac{\Omega H}{N} \sin \frac{i \Omega H}{N}
$$

Applying (28) with Taylor series expansion for the coefficient terms " $\Omega H$ ", we obtain

$$
\begin{aligned}
& \Delta \widehat{V}_{\text {sculm }}=b c H\left\{\left[k_{1}+2 k_{2}+\cdots(N-1) k_{N-1}\right]\right. \\
& \cdot\left(\frac{\Omega H}{N}\right)^{2} \\
& +\left(-\frac{k_{1}}{3 !}-\frac{k_{1}}{3 !}-\frac{2 k_{2}}{3 !}-\frac{2^{3} k_{2}}{3 !}-\frac{3^{3} k_{3}}{3 !}-\frac{3 k_{3}}{3 !} \cdots\right) \\
& \left.\quad\left(\frac{\Omega H}{N}\right)^{4}+\ldots\right\}
\end{aligned}
$$

Applying (15) with Taylor series expansion for the coefficient terms " $\Omega H$ " gives

$$
\Delta V_{\text {sculm }}=\frac{b c}{2}\left[\frac{(\Omega H)^{3}}{3 ! \Omega}-\frac{(\Omega H)^{5}}{5 ! \Omega}+\cdots\right]
$$

From $\Delta \widehat{V}_{\text {sculm }}=\Delta V_{\text {sculm }}$ we can obtain

$$
A_{(N-1) \times(N-1)} \cdot G_{(N-1) \times 1}=D_{(N-1) \times 1}
$$

where

$$
A=\left[\begin{array}{cccc}
\frac{1}{N^{2}} & \frac{2}{N^{2}} & \cdots & \frac{N-1}{N^{2}} \\
\frac{1+1^{3}}{N^{4} 3 !} & \frac{2+2^{3}}{N^{4} 3 !} & \cdots & \frac{(N-1)+(N-1)^{3}}{N^{4} 3 !} \\
\cdots & \cdots & \cdots \\
\frac{1+1^{(2 N-3)}}{N^{2(N-1)}(2 N-3) !} & \frac{2+2^{(2 N-3)}}{N^{2(N-1)}(2 N-3) !} & \cdots & \frac{(N-1)+(N-1)^{(2 N-3)}}{N^{2(N-1)}(2 N-3) !}
\end{array}\right],
$$




$$
\begin{aligned}
& G=\underbrace{\left[\begin{array}{llll}
k_{1} & k_{2} & \cdots & k_{N-1}
\end{array}\right]^{T},}_{(N-1) \times 1} \\
& D=\underbrace{\left[\begin{array}{llll}
\frac{1}{2 \cdot 3 !} & \frac{1}{2 \cdot 5 !} & \cdots & \frac{1}{2 \cdot(2 N-1) !}
\end{array}\right]^{T}}_{(N-1) \times 1}
\end{aligned}
$$

The solution to (31) is $G=A^{-1} D$. Details regarding the optimal coefficients are shown in Table 1.

Comparing the developed sculling algorithm given by (22) with the conventional sculling algorithms represented in (5) or (7), we can see the advantages of the developed algorithm are that it is able to calculate out the sculling correction and then the velocity at $t_{m}$ directly without any requirements to perform the dimension conversion of inertial sensor outputs.

\section{Sculling Algorithm Using Angular Rate/Specific Force Increments Input}

4.1. Generalized Sculling Algorithm. If inertial sensors output angular rate/specific force increments, for a 2-interval sculling velocity algorithm there are three sample gyro outputs $\omega(i=1,2,3)$ at times $t_{m-1}, t_{m-1 / 2}$, and $t_{m}$ and two sample accelerometer outputs $\Delta V_{j}(j=1,2)$ at times $t_{m-1 / 2}, t_{m}$ over an update period $\left(t_{m-1}, t_{m}\right)$. Assume that the angular rate $\omega$ and the specific force increments $\Delta V_{j}$ can be approximated by the second-order polynomial:

$$
\begin{aligned}
\omega & =a+2 b\left(t-t_{m-1}\right)+3 c\left(t-t_{m-1}\right)^{2} \\
\Delta V & =A\left(t-t_{m-1}\right)+B\left(t-t_{m-1}\right)^{2}
\end{aligned}
$$

where $a, b, A, B$, and $C$ are the polynomials coefficients. We then obtain the following:

$$
\begin{aligned}
A H & =3 \Delta V_{1}-\Delta V_{2} \\
B H^{2} & =2\left(\Delta V_{2}-\Delta V_{1}\right), \\
a & =\omega_{1} \\
b H & =\frac{1}{2}\left(-3 \omega_{1}+4 \omega_{2}-\omega_{3}\right) \\
c H^{2} & =\frac{2}{3}\left(\omega_{1}-2 \omega_{2}+\omega_{3}\right)
\end{aligned}
$$

Comparing (9) and (10) with (33) and (34) we can see that there is a duality between the sculling algorithm using incremental angle/specific force inputs and the sculling algorithm using angular rate/specific force increments inputs. Eqs. (9) and (10) have the identical mathematical forms to (33) and (34), and they equal each other when the terms $\Delta \theta$, $f, a H, b H^{2}, A, B H$, and $C H^{2}$ in (9) and (10) are replaced by $\Delta V, \omega, A H, B H^{2}, a, b H$, and $c H^{2}$ respectively. The 2 -interval generalized sculling algorithm using angular rate/specific force increments inputs can therefore be given as

$$
\begin{aligned}
\Delta \widehat{V}_{\text {sculm }}= & \frac{H}{20} \Delta V_{1} \times \omega_{1}-\frac{7 H}{60} \Delta V_{2} \times \omega_{1}+\frac{4 H}{15} \Delta V_{1} \\
& \times \omega_{2}-\frac{4 H}{15} \Delta V_{2} \times \omega_{2}+\frac{7 H}{60} \Delta V_{1} \times \omega_{3} \\
& +\frac{H}{20} \Delta V_{2} \times \omega_{3}
\end{aligned}
$$

4.2. Formalized Optimal Sculling Algorithm. For $N$-interval sculling velocity algorithm using angular rate/specific force increments inputs, the sample number of the accelerometer outputs (specific force increments) is $N-1$, and the sample number of the gyro outputs (angular rate) is $N$. The gyro/accelerometer outputs in a sculling environment defined by (13) are

$$
\begin{aligned}
& \omega_{i}=b \Omega \cos \left[\Omega\left(t_{m}+\frac{i-1}{N} H\right)\right] J, \\
& i=1,2, \ldots, N+1 \\
& \Delta V_{j}=\frac{2 c}{\Omega} \sin \frac{\Omega H}{2 N} \sin \Omega\left[t_{m-1}+\frac{(2 j-1) H}{2 N}\right] K, \\
& j=1,2, \ldots, N
\end{aligned}
$$

Similar to the derivations of (15)-(17), the generalized form for sculling correction algorithm using angular rate/specific force increments inputs is

$$
\begin{array}{r}
\Delta \widehat{V}_{\text {sculm }}=\sum_{j=i+1}^{N} \sum_{i=1}^{N-1} k_{i j}\left(\omega_{i} \times \Delta V_{j}-\omega_{j} \times \Delta V_{i}\right) H, \\
\quad N=2
\end{array}
$$

It can be derived from (36) that

$$
\begin{aligned}
& \left(\omega_{i} \times \Delta V_{j}-\omega_{j} \times \Delta V_{i}\right) H \\
& =b c H \sin \frac{\Omega H}{2} \sin \left(\frac{j-i}{2} \Omega H\right)
\end{aligned}
$$

Obviously, the sculling correction $\Delta \widehat{V}_{\text {sculm }}$ in (37) is only controlled by $|j-i|$. Therefore (37) can be simplified as

$$
\Delta \widehat{V}_{s c u l m}=\sum_{j=2}^{N} k_{j-1}\left(\omega_{1} \times \Delta V_{j}-\omega_{j} \times \Delta V_{1}\right) H
$$




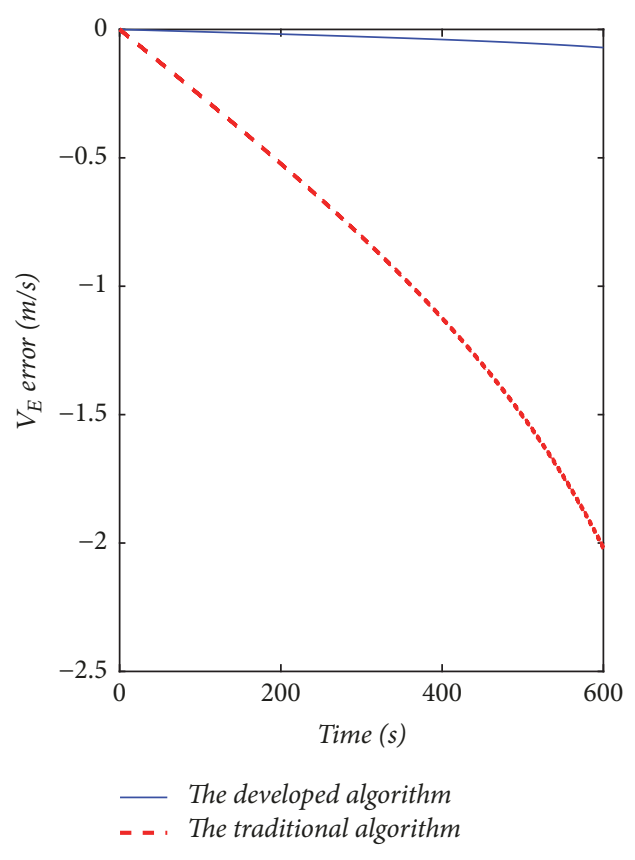

(a) Eastward velocity error

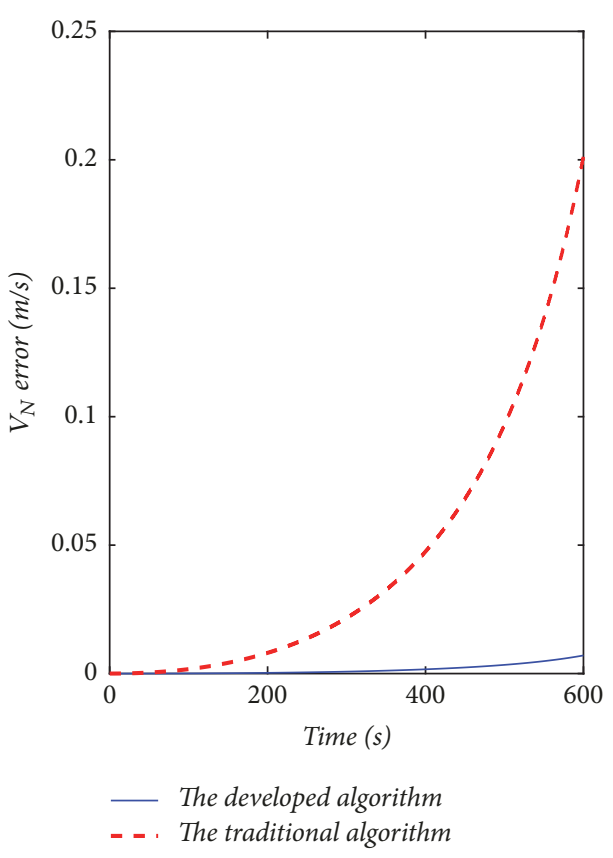

(b) Northward velocity error

FIGURE 1: Comparison of the horizontal velocity determination results between two algorithms under sculling environment.

TABLE 1: Sculling algorithm optimal coefficients.

\begin{tabular}{lcc}
\hline$N$ & $G=A^{-1} D$ & $\delta \widehat{V}_{\text {sculm }}$ (per-unit algorithm error) \\
\hline 2 & $k_{1}=\frac{1}{3}$ & $\frac{b c}{360}(\Omega H)^{4}$ \\
3 & {$\left[\begin{array}{l}k_{1} \\
k_{2}\end{array}\right]=\left[\begin{array}{ll}\frac{23}{40} & \frac{7}{80}\end{array}\right]^{T}$} & $\frac{17 b c}{4898880}(\Omega H)^{6}$ \\
$\ldots$ & $\ldots$ & $\ldots$ \\
\hline
\end{tabular}

Eqs. (19) and (39) have the identical mathematical forms when the terms of $\Delta \theta, f$ in (19) are replaced by $\Delta V, \omega$. Hence the optimal values for coefficients $k_{1}, k_{2} \ldots$ in (39) are as same as those in (9). That is, the optimal values for coefficients shown in Table 1 are also appropriate for the sculling algorithm using angular rate/specific force increments inputs. For example, the optimal coefficient for a 2-interval sculling algorithm using angular rate/specific force increments inputs is $k=1 / 3$, and the corresponding sculling algorithm is

$$
\Delta \widehat{V}_{s c u l m}=\frac{1}{3}\left(\omega_{1} \times \Delta V_{2}-\omega_{2} \times \Delta V_{1}\right) H
$$

The algorithm error per unit time is

$$
\delta \widehat{V}_{\text {sculm }}=\left|\widehat{V}_{\text {sculm }}-V_{\text {sculm }}\right|=\frac{b c}{360}(\Omega H)^{4}
$$

\section{Simulations}

The sculling motion is defined by (13). For this 600s duration test, the amplitude of the angular vibration $b=1^{\circ}$, the amplitude of the specific force vibration $c=10 g$, the frequency associated with the angular and specific force oscillations $\Omega=2 \pi \mathrm{rad} / \mathrm{s}$ (oscillation frequency is $1 \mathrm{~Hz}$ ). The inertial sensors outputs are given by (16). The velocity update period $H=0.01$ s. The initial attitude is $(0,0,0)^{\circ}$. The initial velocity is $(0,0,0) \mathrm{m} / \mathrm{s}$. The initial position is $\left(118.813773^{\circ}, 32.035512^{\circ}\right.$, $10 \mathrm{~m}$ ). The navigation coordinate frame is set to east-northup. The error comparisons between the developed 2-interval sculling algorithm given by (22) and the conventional 2interval sculling algorithm given by (5) are shown in Figures 1 and 2 . The solid line represents the developed sculling algorithm errors; the dotted line represents the conventional sculling algorithm errors.

As can be seen in Figure 1, the eastward velocity errors of both algorithms are larger about one order than those northern velocity errors. This is because in a sculling environment defined by (13), the sculling error mainly exists in the $x$-axis of the carrier. The corresponding velocity component is $V_{\mathrm{E}}$ in the east-north-up coordinate frame.

Particularly, for the traditional algorithm the sculling correction term $\Delta V_{\text {sculm }}$ given in (15) has not been compensated effectively due to the computational error caused by the conversion of specific force into specific force increments by digital integration. There is a constant error term in the eastward velocity of the traditional algorithm, so the eastward velocity error curve of the traditional algorithm increases rapidly and linearly due to the error propagation.

However, the accuracy of both $V_{\mathrm{E}}$ and $V_{N}$ as estimated by the proposed algorithm is improved by one order of magnitude when compared with those measured by the traditional sculling algorithm. This is because using the proposed sculling algorithm can calculate out the velocity directly without dimension conversion of inertial sensor 


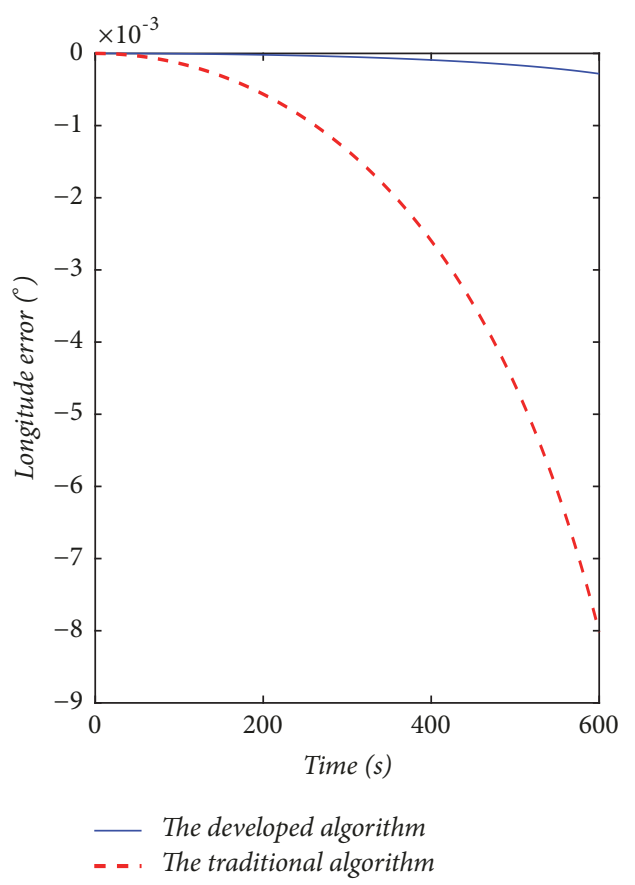

(a) Longitude error

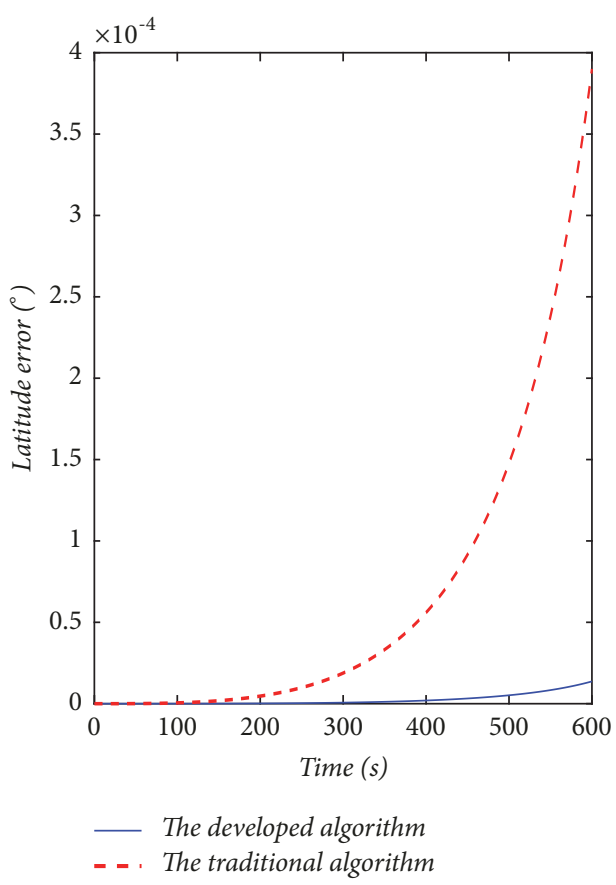

(b) Latitude error

FIGURE 2: Comparison of the horizontal position determination results between two algorithms under sculling environment.

outputs. Therefore, the sculling correction has been compensated effectively and the velocity determination precision is improved dramatically.

From Figure 2 we can see that the longitude errors of both algorithms are larger than the latitude errors of both algorithms. This is because the eastward velocity error is the main reason for the longitude error. As mentioned in the third paragraph before, the eastward velocity errors of both algorithms are larger about one order than those northern velocity errors.

The increase of the longitude error curve of the traditional algorithm is particularly rapid, because when the inertial sensors output integrated angular rate/specific force and the carrier is in a sculling motion environment, the traditional algorithm cannot compensate the sculling correction well. It will induce a rapid error propagation in eastward velocity and then a rapid increasing longitude error finally.

However, the position errors of the proposed algorithm are reduced by more than one order of magnitude compared with those of the traditional sculling algorithm. This is because the improved precision in velocity determination will also result in improved precision in position determination.

\section{Trial Study}

As is shown in Figure 3, a dynamic car test was performed to validate the performance of our proposed sculling algorithm. For this 20 -minute test, the constant gyro drift is $0.01^{\circ} / \mathrm{h}$; the accelerometer bias is $5 \times 10^{-5} \mathrm{~g}$. The update period for the velocity algorithm is $0.01 \mathrm{~s}$. A GPS/INS integrated navigation system (SPAN-CPT, NovAtel in Sweden) is also used as the benchmark for attitude, velocity, and position. NovAtel has

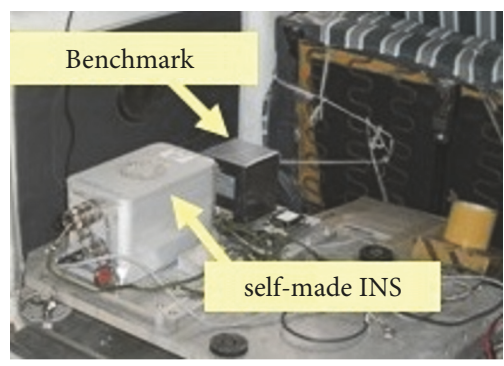

FIGURE 3: Experimental photograph.

claimed that the performance of this integrated navigation system is better than $0.06^{\circ}$ for heading and $0.02^{\circ}$ for pitch and roll, $0.015 \mathrm{~m} / \mathrm{s}$ for horizontal velocity, and $1.2 \mathrm{~m}$ for horizontal position accuracy.

Trial results for the comparative study between the novel and traditional algorithm are shown in Figures 4 and 5. Obviously, the accuracy of horizontal (eastward/northward) velocity and horizontal position (longitude/latitude) is improved when using the novel algorithm. These trial results are consistent with both theoretical analysis (Section 3.3) and simulation results before (Figures 1 and 2).

\section{Conclusions}

To solve the issue that in certain situations the output parameters of inertial sensor do not match the input parameters required by the conventional sculling algorithms, an optimal sculling algorithm using incremental angle/specific force input or angular rate/specific force increments inputs is 


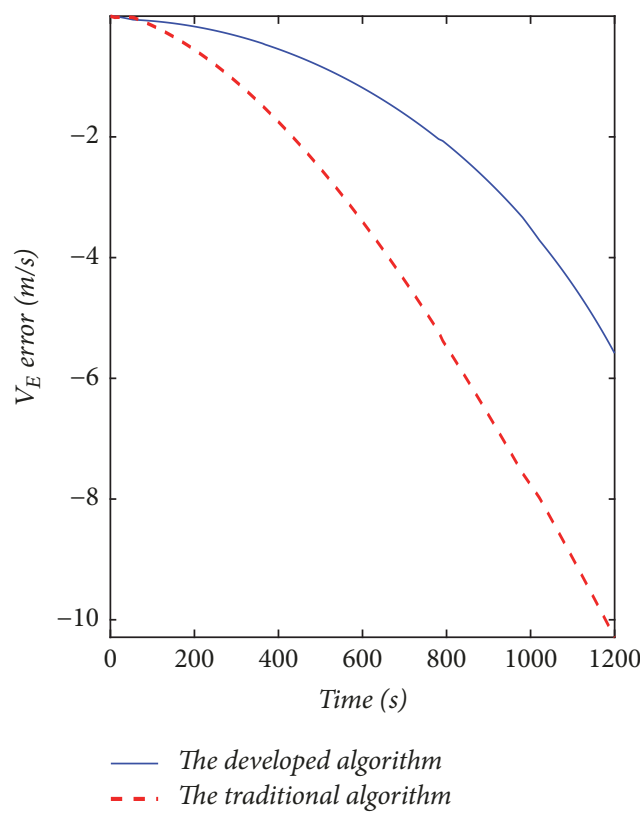

(a) Eastward velocity error

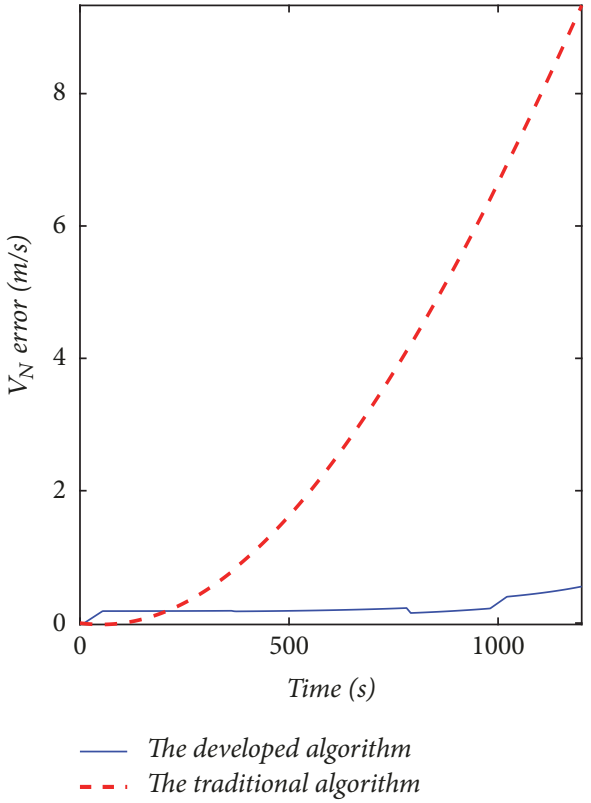

(b) Northward velocity error

FIGURE 4: Comparison of horizontal velocity determination results of the dynamic car test between two algorithms.

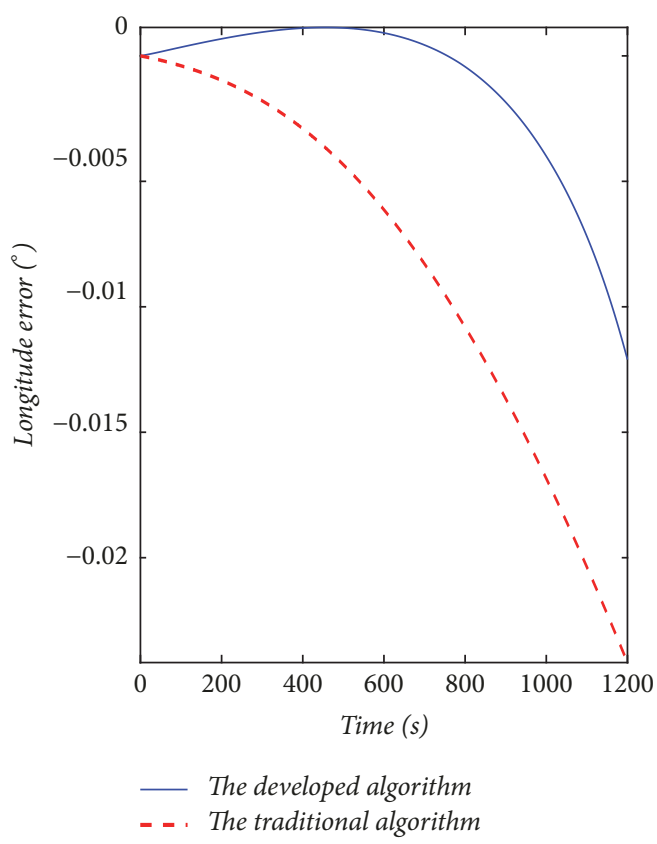

(a) Longitude error

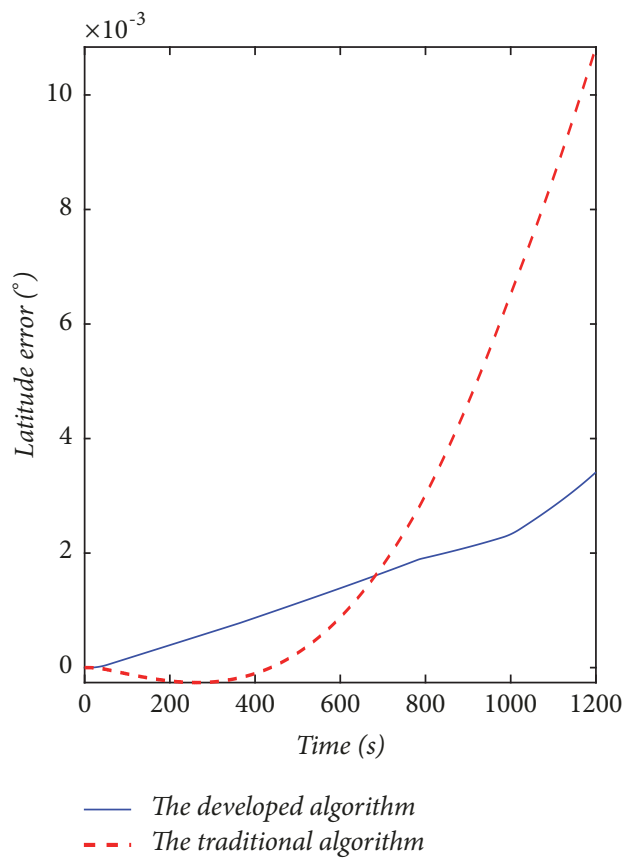

(b) Latitude error

FIGURE 5: Comparison of horizontal position determination results of the dynamic car test between two algorithms.

developed in this paper. The developed sculling algorithms can calculate out the carrier velocity directly without the dimension conversion of inertial sensor outputs. Accordingly, the developed algorithms provide higher precision than conventional sculling algorithms in the SINS in which the employed inertial sensors have the integrated angular rate/specific force or angular rate/specific force increments outputs. Our novel sculling velocity algorithm thus has great useful value in such cases.

\section{Nomenclature}

$f:$ Specific force measured by accelerometers $C_{b}^{n}$ : Direction cosine matrix 
$H$ : Update period

$\triangle \theta_{i}$ : Incremental angle vector over the ith subminor interval

$\omega$ : Angular velocity vector.

\section{Data Availability}

The readers can access the data underlying the findings of the study by visiting https://fairsharing.org/bsg-p000118.

\section{Conflicts of Interest}

The authors declare that they have no conflicts of interest.

\section{Acknowledgments}

The authors would like to acknowledge postgraduate student Di Sang for his work in the dynamic car test. We also appreciate the assistance of the Information Navigation and Measurement and Control Technology Institute of Southeast University, Nanjing, in the use of their equipment. The authors gratefully acknowledge the helpful comments and suggestions of English editors and reviewers. This work was supported in part by the National Natural Science Foundation of China under Grant Nos. 61601228 and 51305207, the Natural Science Foundation of Jiangsu Provincial College under Grant No. 13KJB460010, the Natural Science Foundation of Jiangsu under Grant No. BK20161021, and the Top-Notch Academic Programs Project of Jiangsu Higher Education Institutions (TAPP: PPZY2015A062). This work was also supported by the National Key Research and Development Program of China (Grant No. 2017YFB1103200).

\section{References}

[1] P. G. Savage, "Strapdown inertial navigation integration algorithm design part 2: velocity and position algorithms," Journal of Guidance, Control, and Dynamics, vol. 21, no. 2, pp. 208-221, 1998.

[2] P. G. Savage, "Strapdown sculling algorithm design for sensor dynamic amplitude and phase-shift error," Journal of Guidance, Control, and Dynamics, vol. 35, no. 6, pp. 1718-1729, 2012.

[3] P. G. Savage, Digital Integration Algorithm Error For Random Process Inputs, 2016, Accessed 10 August 2017, http://www .strapdown-associates.com.

[4] M. B. Ignagni, "Duality of optimal strapdown sculling and coning compensation algorithms," NAVIGATION: Journal of the Institute of Navigation, vol. 45, no. 2, pp. 85-95, 1998.

[5] K. M. Roscoe, "Equivalency between strapdown inertial navigation coning and sculling integrals/algorithms," Journal of Guidance, Control, and Dynamics, vol. 24, no. 2, pp. 201-205, 2001.

[6] Y. A. Litmanovich, V. M. Lesyuchevsky, and V. Z. Gusinsky, "Two new classes of strapdown navigation algorithms," Journal of Guidance, Control, and Dynamics, vol. 23, no. 1, pp. 34-44, 2000.

[7] Y. Yu and H. Zhang, "Two interval sculling compensation algorithm based on duality principle," Beijing Hangkong Hangtian Daxue Xuebao/Journal of Beijing University of Aeronautics and Astronautics, vol. 35, no. 3, pp. 326-329, 2009.
[8] Z. Zhang, F. Zhang, and W. Zhang, "Measurement of phase difference for micromachined gyros driven by rotating aircraft," Sensors, vol. 13, no. 8, pp. 11051-11068, 2013.

[9] L. L. Heng, J. Y. Zhang, J. Shen et al., “The improvement and optimization of sculling compensation algorithms for fiberoptical gyro sins," Journal of Projectiles, Rockets, Missiles and Guidance, vol. 31, no. 6, pp. 15-22, 2011.

[10] L. Wang, L. Fu, and M. Xin, "Sculling compensation algorithm for SINS based on two-time scale perturbation model of inertial measurements," Sensors, vol. 18, no. 1, pp. 282-298, 2018.

[11] M. B. Ignagni, "Efficient class of optimized coning compensation algorithms," Journal of Guidance, Control, and Dynamics, vol. 19, no. 2, pp. 424-429, 1996.

[12] L. Xing, Z. Xiong, J.-Y. Liu, and Y.-J. Hang, "Improved Coning and Sculling Error Compensation Algorithms Based on Dual Quaternion for Strapdown Inertial Navigation System," Binggong Xuebao/Acta Armamentarii, vol. 38, no. 7, pp. 1336-1347, 2017.

[13] M. Ignagni, "Optimal Sculling and Coning Algorithms for Analog-Sensor Systems," Journal of Guidance Control Dynamics, vol. 36, no. 3, pp. 903-903, 2015.

[14] Y. Ben, F. Sun, W. Gao, and F. Yu, "Generalized method for improved coning algorithms using angular rate," IEEE Transactions on Aerospace and Electronic Systems, vol. 45, no. 4, pp. $1565-1572,2009$.

[15] C. W. Kang, N. I. Cho, and C. G. Park, "Approach to direct coning/sculling error compensation based on the sinusoidal modelling of IMU signal," IET Radar, Sonar \& Navigation, vol. 7, no. 5, pp. 527-534, 2013. 


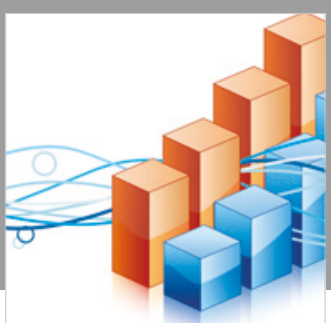

Advances in

Operations Research

\section{-n-m}
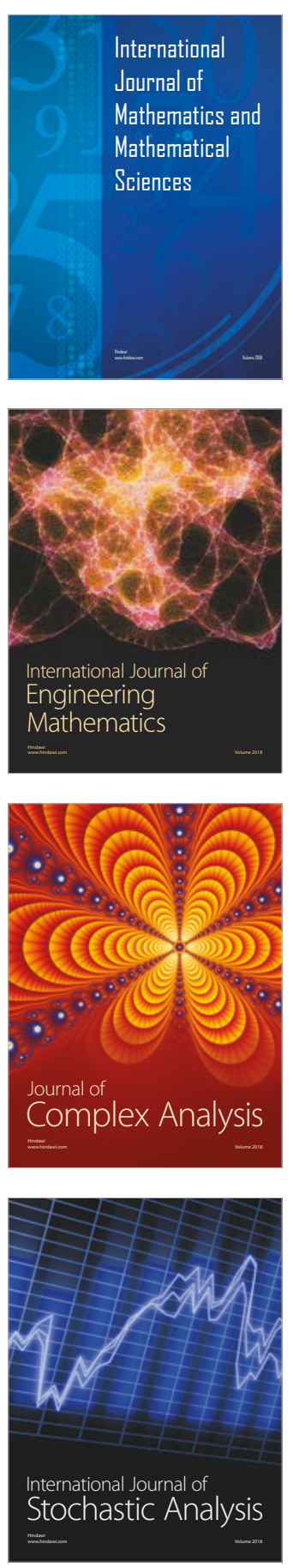
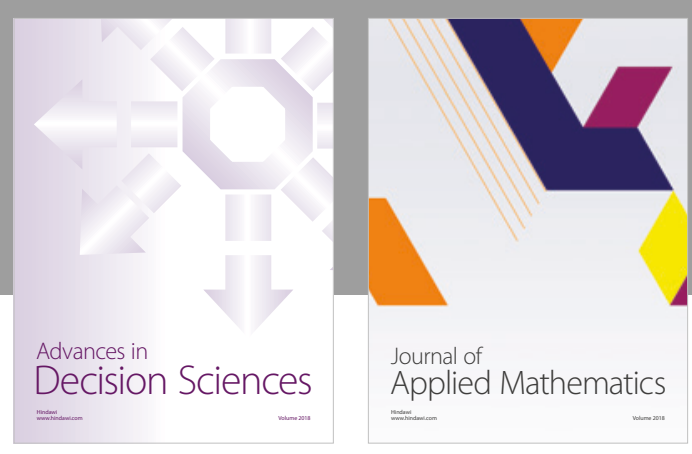

Journal of

Applied Mathematics
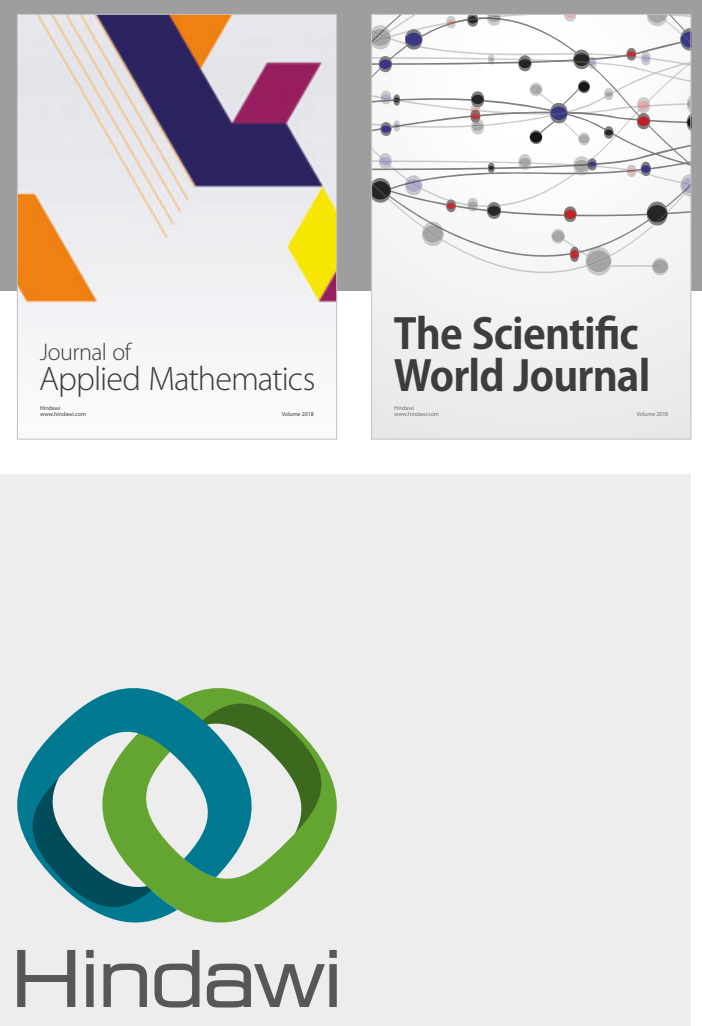

Submit your manuscripts at

www.hindawi.com

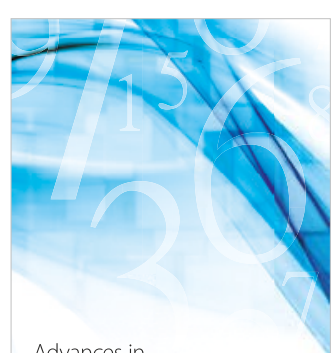

Advances in
Numerical Analysis
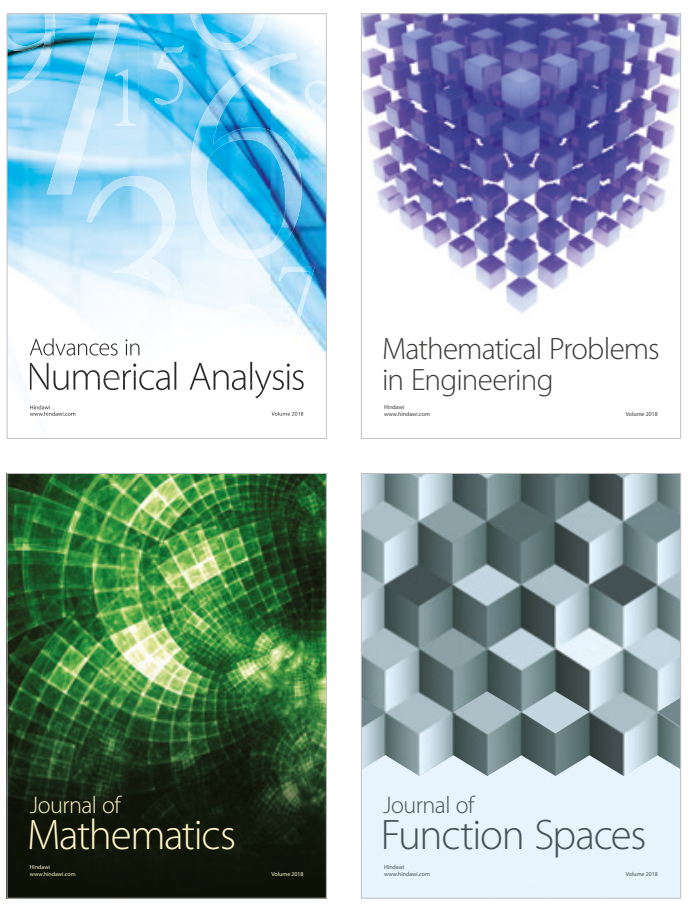

Mathematical Problems in Engineering

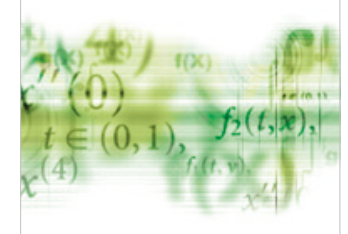

International Journal of

Differential Equations

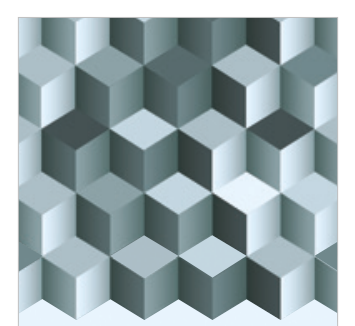

Journal of

Function Spaces

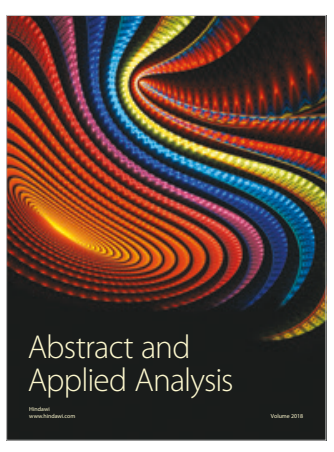

The Scientific

World Journal

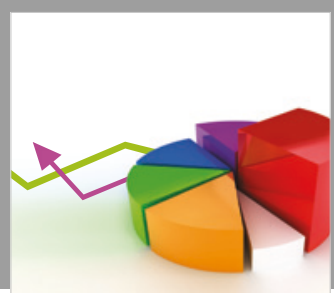

Journal of

Probability and Statistics
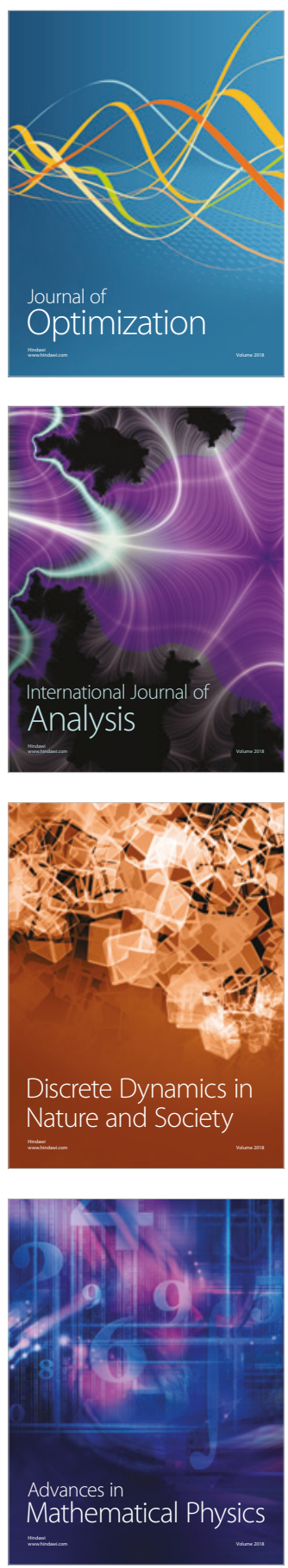